\title{
Biostatistical analysis of quantitative immunofluorescence microscopy
}

images.

Giles $\mathrm{C}^{1,2}$, Albrecht $\mathrm{MA}^{1,2,3}$, Lam $\mathrm{V}^{1,2}$, Takechi $\mathrm{R}^{1,2}$ and Mamo $\mathrm{JC}^{1,2}$.

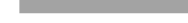

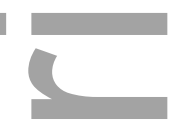

${ }^{1}$ Curtin Health Innovation Research Institute, Curtin University

${ }^{2}$ School of Public Health, Faculty of Health Sciences, Curtin University

${ }^{3}$ Maryland Psychiatric Research Center, School of Medicine, University of Maryland

Corresponding author: Dr. Matthew Albrecht

Email: Matthew.Albrecht@curtin.edu.au

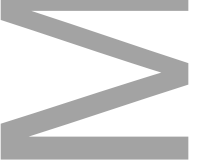

Phone: +1 443-527-1448

School of Public Health, Curtin University

GPO Box U1987

Perth, Western Australia, 6845

Australia

This is the author manuscript accepted for publication and has undergone full peer review but has not been through the copyediting, typesetting, pagination and proofreading process, which may lead to differences between this version and the Version of Record. Please cite this article as doi:

10.1111/1mi.12446.

This article is protected by copyright. All rights reserved.

This is the author manuscript accepted for publication and has undergone full peer review but has not been through the copyediting, typesetting, pagination and proofreading process, which may lead to differences between this version and the Version of Record. Please cite this article as doi:

10.1111/jmi.12446.

This article is protected by copyright. All rights reserved. 


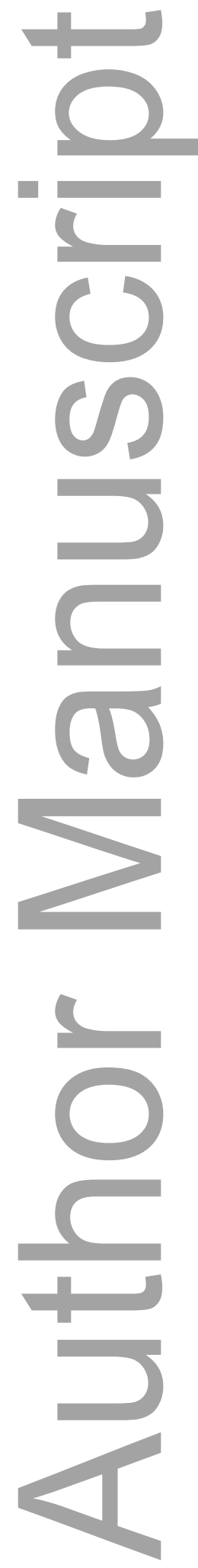

This article is protected by copyright. All rights reserved. 


\section{ABSTRACT:}

Semi-quantitative immunofluorescence microscopy has become a key methodology in biomedical research. Typical statistical workflows are considered in the context of avoiding pseudo-replication and marginalising experimental error. However, immunofluorescence microscopy naturally generates hierarchically structured data that can be leveraged to improve statistical power and enrich biological interpretation. Herein, we describe a robust distribution fitting procedure and compare several statistical tests, outlining their potential advantages/disadvantages in the context of biological interpretation. Further, we describe tractable procedures for power analysis that incorporates the underlying distribution, sample size and number of images captured per sample. The procedures outlined have significant potential for increasing understanding of biological processes and decreasing both ethical and financial burden through experimental optimization.

\section{Keywords:}

Immunofluorescence microscopy, hierarchical analysis, statistics, biological variation.

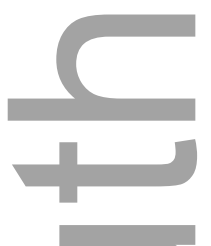

This is the author manuscript accepted for publication and has undergone full peer review but has not been through the copyediting, typesetting, pagination and proofreading process, which may lead to differences between this version and the Version of Record. Please cite this article as doi:

10.1111/mini.12446.

This article is protected by copyright. All rights reserved.

This is the author manuscript accepted for publication and has undergone full peer review but has not been through the copyediting, typesetting, pagination and proofreading process, which may lead to differences between this version and the Version of Record. Please cite this article as doi:

10.1111/jmi.12446.

This article is protected by copyright. All rights reserved. 


\section{INTRODUCTION}

The application of more detailed statistical models to quantitative immunofluorescence data may provide more powerful and accurate characterisation of the effects of interventions compared to the more commonly used analysis of point estimates of central tendency (e.g., mean) (Chinta et al., 2012, Handfield et al., 2015, Lindvere et al., 2013, Yang, 2013). An underutilised feature of immunofluorescence microscopy data is that the experimental designs used naturally generate hierarchical data that can be leveraged to improve statistical analysis (Park et al., 2013, Vallmitjana et al., 2013). In this context, the application of hierarchical (or multi-level) parameter estimation provides a practical and theoretically sound approach to enhancing the stability and efficiency of a statistical analysis (Duncan et al., 1998, Ji \& Liu, 2010). Hierarchical analysis does this by using all of the available data and the structure of the data to apply principled soft constraints on more extreme parameter estimates. Such soft constraints are applied using varying degrees of shrinkage depending on where there is sufficient information from the structure of the hierarchy to be applied to a single parameter estimate, or in situations where there is a lack of data to obtain a stable parameter estimate (i.e., applied relative to the precision of the data). In hierarchical modelling, parameters are-estimated for each experimental unit (e.g., a mean and standard deviation (SD) for each animal assuming a Gaussian distribution) and these parameters are drawn from overarching distributions (e.g., each animal's mean is drawn from the parent distribution) that explicitly model variations between experimental treatments.

A significant disadvantage of commonly utilized analyses is the large number of observations that can be required for each subject, as a small number of images 
collected per sample can impede obtaining stable estimates of subject-level parameters. For immunofluorescence analysis, specimen size or availability, the cost of antibodies, time on microscope, time for image analysis and the storage of exceedingly large datasets can limit data collection. The application of a hierarchical linear model (HLM) notionally allows partial pooling of information across specimens for parameter estimation by assuming that each parameter can be drawn from a parent distribution. Partial pooling increases stability and efficiency by applying shrinkage to parameter estimates according to the hierarchical structure (Greenland, 2000). Shrinkage refers to the property of a parameter of a singular unit (e.g., experimental unit mean) being shifted towards the overall group parameter estimate (e.g., treatment group mean) (Greenland, 2000, Ji \& Liu, 2010). The latter is useful when few images are processed per replicate, by allowing flexible and continuous control of the solution space available to each parameter.

A further limitation when comparing treatment effects on quantitative immunofluorescence image data using a single measure of central tendency (obtained from the compilation of data from multiple images) is that it ignores the potential biological relevance of variability (Handfield et al., 2015, Hyman et al., 1995, Vallmitjana et al., 2013). Taking multiple images in immunofluorescence microscopy results in a better estimate of the subject's 'true-value', i.e., the more images taken, the greater the confidence in closing in on a more accurate estimate of the subject's average level of fluorescence. The differences between images are therefore considered random, or experimental error (albeit, which might possess spatial or structural significance), which is marginalised via averaging within treatment effects. The latter is a valid approach when the errors are normally distributed and measures of centrality are of primary interest. However, a large 
proportion of the measured variance of immunofluorescence images may reflect important biological variation and may be highly informative in a systems physiology context.

A related distributional issue arises during the calculation of power for an immunofluorescence experiment. Sample size and power calculations often do not take into account the interaction between sampling methodology and the statistical distribution of measured values. For example, basing power/sample size calculations on measures of central tendency may not be appropriate for immunofluorescence data of biological specimens when they possess a right-skewed distribution (Hyman et al., 1995, Lampariello \& Aiello, 1998). In addition, given the nature of data collection in a naturally hierarchical structure, the generative distribution/model of immunofluorescence data is better reflected by hierarchical parameter estimation approach when considering power, sample size and treatment effects.

The rationale for the current paper is to elucidate a statistical method that maximises the use of data and reflects the generative structure of the data of the experimental approach when collecting immunofluorescence data in biological systems. Here, we illustrate an improvement in precision and power when using the hierarchical method compared with a conventional statistical analysis. We use empirical data quantifying the abundance of immunoglobulin $\mathrm{G}$ within brain parenchyme used as a surrogate marker of blood-to-brain leakage across capillaries of plasma derived proteins (Takechi et al., 2010). We then contrast two alternate treatment groups of mice to highlight the statistical advantages of the hierarchical method.

\section{METHODS}




\subsection{Animals and intervention}

Female Sprague Dawley rats were purchased from the Animal Resource Centre (ARC; Perth, WA, Australia) and housed in an accredited animal holding facility. The animals were housed on a 12 hour light/dark cycle with ambient temperature 22 degrees Celsius. Following a single week holding phase, animals were supplied ad libitum access to water and their respective diets. The control group was given a low fat maintenance diet (Glen Forrest Stock Feeds; AIN-93G), while the treatment group was supplied a modified control diet to induce capillary permeability (Lam et al., 2015), formulated to deliver 40,000 IU of vitamin D3 per $\mathrm{kg}$ of diet consumed (daily ingested dose of approximately 600 international units (IU) vs 15 IU for control animals).

\subsection{Sample preparation and semi-quantitative immunofluorescence microscopy}

Twelve weeks after initiating the diet, animals were administered an IP dose of ketamine/xylazine (75 mg/kg ketamine; $10 \mathrm{mg} / \mathrm{kg}$ xylazine). Once complete anaesthesia had been achieved, blood was collected through cardiac puncture. Brains were excised, washed in ice cold PBS. A mid sagittal cut was performed, separating the two hemispheres. Both hemispheres were fixed in $4 \%$ paraformaldehyde solution for 24 hours. Following fixation, the hemispheres were cryoprotected for three days in a $20 \%$ solution of sucrose in PBS. Following cryoprotection, the brains were frozen in isopentane/dry ice and stored at -80 degrees Celsius.

Frozen hemispheres (right) were sectioned on a cryostat into 20 micron sections (sagittal sections) and mounted on silane coated slides. Sections were hydrated with 
PBS, followed by non-specific binding sites blocking with $10 \%$ goat serum. Parenchymal lgG was determined as previously described (Takechi et al., 2010). Briefly, Goat anti-rat IgG antibodies conjugated to Alex-Fluor 488 was incubated (1:100 dilution) with the samples for 24 hours at 4 degrees Celsius. Sections were counter stained with DAPI (for nuclear stain) and mounted with anti-fade mounting medium and coverslips.

Stained sections were imaged on a Zeiss microscope, with an ApoTome module for structured light microscopy. A Zeiss Axiocam CCD camera was used for image capture. All images were taken with a $20 \times$ PlanNeo objective (NA 0.5). Zeiss Axiovision 4.8 was used for image capture, with optical sectioning through the ApoTome module.

Images were randomly captured throughout the cortex of each hemisphere section, in three dimensions. Roughly 20-40 images were captured per section. Each image consisted of 8-12 z-stack images (1.25 $\mu \mathrm{M}$ z-steps).

Image analysis was performed using Volocity 6.1. In brief, the densitometric sum of fluorescent intensity was determined for the perivascular spaces that represented the extravasation of IgG. Raw densitometric sums for each image were recorded and used in the statistical analysis (20-40 densitometric sums per animal corresponding to the number of images taken).

\subsection{Determination of statistical distribution}

Herein, we assume the extravasation of $\lg G$ of randomly selected regions throughout the cortex follows a parametric distribution and describe methods to assess the distribution. 
Empirical cumulative density functions (ECDF) are step functions to estimate the underlying cumulative distribution of a sample. We fitted an ECDF for each animal's IgG distribution. Using the Kolmogorov-Smirnov (KS) test, we tested the fit of the following distributions: normal, Weibull and log-normal. As the D statistic obtained through the KS-test cannot be used to test whether the ECDF came from a distribution whose parameters were obtained from the sample, a parametric bootstrap to test goodness-of-fit was adopted (Clauset et al., 2009).

For each animal, the maximum likelihood estimate of parameter values for each distribution of interest was determined. Using these parameters, we generated bootstrap samples per animal. For each bootstrap sample, the maximum likelihood estimate of parameters was determined and the D statistic calculated using the KStest. For each animal/distribution, 10,000 bootstrap samples were generated to obtain a distribution of D-statistics that was used to test goodness-of-fit. The upper $95^{\text {th }}$ percentile (or the $9,500^{\text {th }}$ largest value) was used as the critical value. To calculate a p-value for goodness of fit, the number of D-statistic values that were higher than the D-statistic we calculated in the original dataset and divided by the number of bootstrap replicates. A low p-value indicates that there is evidence to indicate that the sample did not come from that the tested distribution. Typical values used are a p-value of 0.05 , as was used in this demonstrative analysis; however, a more conservative value of 0.1 could be adopted. Following the identification of candidate distributions that fit the data, the Akaike Information criterion (AIC) was further used to contrast distributions. AIC reduces over-fitting by penalising models with a greater number of free parameters.

\section{$2.4 \quad$ Hierarchical Model}


Following identification of the best fitting model, we were then able to construct the hierarchical model. In brief, each subject's distribution of IgG extravasation was assumed to be drawn from a parent distribution (Figure 1). For each subject-level parameter in the model, an appropriate higher-level distribution must be described for that parameter. We used maximum likelihood estimation to estimate a set of parameters that maximises the probability of observing a given dataset. In the case of a normal distribution, two parameters yield the maximum likelihood estimate: $\mathrm{mu}=$ mean of the sample; sigma = population standard deviation. The likelihood function (joint probability function) was constructed as follows: for animal $j$, from group $i$, the probability density function of densitometric sum for image $k$ is given by

$$
p\left(y_{i j k} \mid \mu_{i j}, \phi_{i j}\right)=\frac{e^{\frac{-\left(n n y_{i j k}-\mu_{i j}\right)^{2} \phi_{i j}}{2}} \sqrt{\phi_{i j}}}{y_{i j k} \sqrt{2 \pi}}
$$

Where $\mu_{i j}$ and $\phi_{i j}$ are the mean and precision (1/variance) of the log-normal distribution. Parent distributions for $\mu_{i j}$ and $\phi_{i j}$ were assumed to be Gaussian and gamma distributions, respectively.

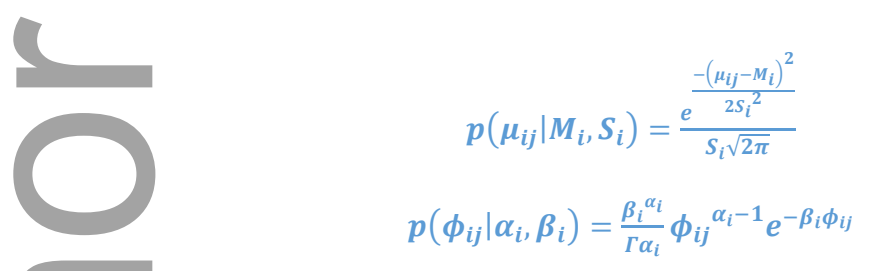

A joint likelihood function was then defined for the parent distribution parameters as follows:

$$
L\left(\Theta \mid y_{i}\right)=\prod_{i=1}^{P} \iint p\left(y_{i j k} \mid \mu_{i j}, \phi_{i j}\right) p\left(\mu_{i j} \mid M_{i}, S_{i}\right) p\left(\phi_{i j} \mid \alpha_{i}, \beta_{i}\right) d \mu_{i j} d \phi_{i j}
$$

where $\Theta$ is the parent distribution parameters $\left[M_{i}, S_{i}, \alpha_{i}, \beta_{i}\right]$ and $P$ is the number of animals within a group. With the given function, the population parameters were estimated by minimising the negative log-likelihood (equivalent to maximising the likelihood). Once the maximum likelihood parent distribution parameters were 
generated, the modal estimates for animal level distributions were determined by maximising for each animal:

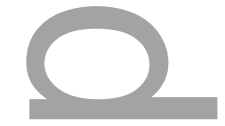

$\widehat{\mu, \sigma}=\arg \max _{\mu, \sigma} f(x \vee \mu, \phi) f(\mu \vee M, S) f(\phi \vee \alpha, \beta)$

\subsection{Statistical test}

Null and alternative hypotheses for a two-group comparison were constructed for a likelihood ratio test.

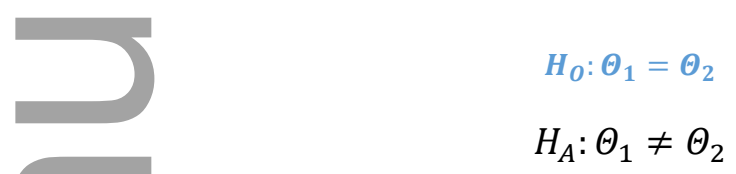

Where $\Theta=[M, S, \alpha, \beta]$. This approach constitutes a nested test. The distribution of a nested likelihood ratio test statistic (D) asymptotically approaches a chi-square distribution with degrees of freedom equal to the difference in dimensionality (number of parameters constrained).

$$
D=-2 \ln \frac{L\left(\text { nullhypothesis } \mid y_{i j k}\right)}{L\left(\text { alternativehypothesis } \mid y_{i j k}\right)}
$$

A parametric bootstrap was used to estimate the test statistic distribution. Synthetic data generated under the null hypothesis was used to obtain the test statistic distribution for comparison with the chi-square distribution. An example of the generative procedure is shown in Figure 1. Using the maximum likelihood parent distribution parameters obtained under the null hypothesis, 10,000 datasets were generated with identical animal and image numbers as collected in the real animal study.

\subsection{Power analysis}


Sample size and power analyses were done by Monte Carlo simulation. We used a realistic sampling method (see Figure 1), simulating data obtained with a given sample size (range $=4-12$ ), image number (range $=5-80)$, parent distribution parameter set and statistical test (t-test, hierarchical, etc.). For each of these simulated data sets, 10,000 bootstrap samples were taken to perform statistical inference. Effect sizes were simulated by assigning to one group parameters from the parent distribution of the control group $(M=13.70 ; S=0.51$; alpha=12.93; beta=35.95), while the other group's parent distribution parameters ( $M$ and $S$; alpha and beta; or all) were progressively translated further from the control group (Table 1). Gamma distribution parameters were generated based on translation of $S D$, followed by conversion into precision.

These simulations enabled us to compare the performance of hierarchical maximum likelihood method against traditional approaches for statistical analysis (usage of arithmetic mean of densitometric sum). Type I and II errors for each statistical method is recorded. For all t-tests performed in this paper, the t-test was performed with the (often incorrect) assumption of equal variances in order to reflect an indiscriminate application of statistical tests.

\subsection{Computation}

All statistical procedures were custom written in C++ (Visual Studio 2013). A custom Nelder-Mead simplex algorithm was used as the optimization routine for minimising the negative log likelihoods. The two dimensional integration in the joint likelihood equation was calculated using 32 point Legendre quadrature for each parameter. The limits of the integration covered the percentiles from 0.01 to 0.99 of the distribution. 


\section{RESULTS}

From each animal ( 2 groups with 8 per group, total $N=16$ ), we obtained $20-40$ immunofluorescence microscopic images randomly from the cortex. Each image contained between 8-12 z-stack images. The densitometric sum of extravascular IgG fluorescence was determined for each image. A compilation of animal ECDF and example immunofluorescence micrographs are shown in Figure 2.

\subsection{Distributional analysis}

Figure 2 presents the $\lg G$ empirical cumulative density functions for each animal. The distributions fitted were: normal, weibull and log-normal. Figure 2 presents boxplots of the D-statistic from the distribution tests. Table 2 presents the bootstrapped $p$-values for each animal's distribution fit test and AIC value. The best fitting distribution was the log-normal distribution. Therefore, for the hierarchical analysis, only the log-normal distribution is presented.

\subsection{Hierarchical Analysis}

Hierarchical models for both the null hypothesis and the alternative hypothesis were fitted. The maximum likelihood estimates of parent distribution parameters and negative log likelihoods for each hypothesis are shown in Table 3. The likelihood ratio statistic contrasting the null and alternative models was 13.2 , suggesting that the alternative model was a better fit to the data indicating an effect of treatment/Vitamin D on IgG extravasation.

The superiority of the alternative model was statistically formalised by obtaining 10,000 boot strapped estimates of the likelihood ratio statistic. Stratified bootstrap 
samples were obtained following the sampling structure outlined in Figure 1. Figure 3 shows the resultant distribution of likelihood ratio statistics along with a chi-square distribution for comparison (degrees of freedom $=4$ ). As can be seen from the figure the chi-square distribution follows very closely the empirical distribution and is therefore appropriate for obtaining critical values for statistical inference. For the bootstrapped samples an alpha of 0.05 was associated with a critical value of 9.7 , compared to a critical value of 9.5 using the chi-square distribution. Due to the test statistic being larger than the critical value, we reject the null hypothesis.

Following determination of the parent distribution parameters, we estimated the individual level parameters (under both the null and alternative hypothesis for demonstrative purposes) by finding the modal estimates described above. Figure 4 shows the estimates with corresponding 95\% confidence intervals. For contrast, animal densitometric sums (mean/SD + 95\% Cl) following log-transformation and on the untransformed scale are shown in Figure 5.

\subsection{Simulated type I error rates}

Following the distributional analysis, log-normal data were generated using the structure of the experimental procedure with respect to simulating nested images in animals (Figure 1). For the type I error analysis, we used the control animal parent distribution parameters (i.e., $M=13.70, S=0.51$, alpha=12.93, beta=35.95) assuming no difference between parent distributions between the treatment and control groups. We contrasted the type I error rate of the hierarchical procedure with analyses using the standard parametric t-test on the original data, a t-test on the logtransformed data, and a non-parametric analysis Mann-Whitney $U$ analysis on subject level means within treatment groups on the original scale of the data. 
Table 1 shows that the proportion of type I errors (false positives) was below 0.05 for both the t-test and Mann-Whitney $U$ test, indicating that these tests are conservative for this experimental design. This is unsurprising, as the t-test is considered to be conservative for non-normal data and particularly when the number of experimental replicates are low ( $n=16$ in this study). The t-test on log-transformed data recorded a proportion of 0.051 , slightly over the nominal alpha rate of 0.05 . The hierarchical maximum likelihood test performed at the nominal alpha rate of 0.05 when using the bootstrapped critical value, whereas the chi-square critical value results in a slightly less conservative type I error of 0.056 (Table 1 ).

\subsection{Type II error rate and power}

For determination of type II error rates, animal IgG extravasation data was simulated using the control parent distribution parameters and the parameters outlined in Table 1 as the experimental group, contrasting effects across a range of effect sizes. Each parameter set consisted of 10,000 comparisons of simulated datasets. Type I and II error rates are shown in Table 1. For changes in log-normal mean, the t-test on log transformed data achieved the greatest power, followed by the hierarchical maximum likelihood test $>$ Mann-Whitney $U>$ t-test on untransformed data. When there was an increase in log-normal standard deviation, the hierarchical maximum likelihood test was the most powerful, followed by Mann-Whitney $U$ >> t-test on untransformed data. The t-test on log-transformed data was insensitive to changes in standard deviation, maintaining a power close to 0.05 . When the log-normal mean was decreased, but the standard deviation increased, the hierarchical maximum likelihood test was the only test sensitive to detect differences. The t-test on logtransformed data was sensitive to the decrease in the $M$ parameter but not to the increase in animal SD i.e. the t-test on log-transformed data identified the treatment 
group to be less than the control group, despite the expected mean of the (untransformed) treatment group being larger (i.e., the t-test on log-transformed data yielded a significant number of sign errors). This phenomenon has been discussed previously in the literature (Manning \& Mullahy, 2001, Zhou et al., 1997).

To consider the possible effects of image number per animal on the power of the alternate tests, simulated data were generated using the alternative hypothesis parent distribution parameters with a fixed effect size set at 2.45 , but with alternative numbers of images and animals (Table 4). Three-dimensional plots showing the power of each test at each combination of image number and animals is shown in Figure 6. Given that the differences in parameters are primarily mediated through increased log-normal mean, the t-test on log-transformed data consistently outperformed all other statistical tests. Power analyses were most favourable for the HLM model compared to the other models when there was a large number of images per animal, but a small number of animals. By contrast, the non-parametric test performed better than the hierarchical model only when there was a small number of images per animal. The t-test on the untransformed data was consistently the poorest performer.

\section{DISCUSSION}

We describe the application of hierarchical modelling to semi-quantitative immunofluorescence microscopy data combined with a robust distribution fitting approach (Clauset et al., 2009). First, we show that immunofluorescence data is best fit with a log-normal distribution and an appropriate data transformation results in a superior analysis. Second, we show that HLM possesses excellent type I and II 
error properties, but more significantly, the method provides a unified framework for a more detailed consideration of biological effect. That is, the HLM method is able to simultaneously consider differences in both location and scale, two important physiological dimensions.

\subsection{Data Distribution}

We present evidence demonstrating that plasma protein extravasation across cerebral capillary vessels is not normally distributed. From a statistical perspective, treating extravasation data as though it were normally distributed results in a less optimal statistical analysis, increasing Type I and II error rates. The latter is to be expected as when parametric statistics are used, one of the assumptions is that the residuals come from a particular distribution and the tests are optimised for these particular circumstances, e.g., a normal distribution is assumed for the t-test. As described in the results, the non-parametric Mann-Whitney U-test outperforms the ttest in circumstances where the assumptions of the t-test are violated. By contrast, an appropriate transformation significantly enhances the statistical properties of the t-test. Therefore, we and others as a general statistical consideration; (Fagerland \& Sandvik, 2009, Kitchen, 2009, MacDonald, 1999, Parkin, 1993, Zimmerman, 1995) recommend that whenever quantitative immunofluorescence is done with multiple images per specimen, the underlying distribution should be assessed.

\subsection{Biological Variance}

While transformation to a normal distribution can stabilise the measure of central tendency estimate (Figure 5), the potential effects when interpreting biological variance should be considered. For example, ill conceived 'outliers' may represent an extraordinary response to treatment and have significant physiological 
importance. As illustrated in this study, extreme values are evidence of extraordinary abundance of extravasated plasma IgG within focal areas of the cortex. The biological significance of focal areas of significantly compromised cerebral capillary integrity is undoubtedly important. Therefore, interpretation may be confounded if central tendency analysis on the untransformed data is solely adopted, particularly when a large response is 'diluted' by many more 'minor' increased permeability events. In this study, it was expected that there would be large within-subject variance demonstrated by an increase in large leakage sites alongside regions without leakage and which would be amplified by the treatment. However, a review of the data indicated a dramatic shift in image values across all quantiles, suggesting a level of compromised capillary integrity across all vessels.

A concern with data transformations is that tests of central tendency may have different meaning on the transformed scale compared to the untransformed scale. Transforming the log transformed mean back to the original scale (geometric mean) does not give the same estimate as averaging the data on the original scale (mean). Instead, averaging log transformed log-normal data estimates the distribution's overall mean parameter mu. If the data are log-normally distributed, transforming this mu estimate back to the original scale estimates the distribution median $\left(\mathrm{e}^{\wedge} \mathrm{mu}\right)$, whereas averaging on the original scale estimates the distribution mean $\left(e^{\wedge}(m u+v a r / 2)\right)$. This can be seen in the type II power analysis (Table 1); the t-test on log-transformed data tests for equality of the mu parameter, whereas the t-test on untransformed data tests for equality of means $\left(e^{\wedge}(m u+v a r / 2)\right)$. This can confound interpretation if a researcher is unfamiliar with how transformation may influence the test, particularly because the test on transformed and untransformed scale can give different results in significance, effect size and sign. 


\subsection{Hierarchical modelling of the data}

Immunofluorescence microscopy generates hierarchical data, whereby multiple, spatially distributed images are collected from each specimen. In a typical contemporary workflow, quantitative data derived from images are averaged within animals and then statistically analysed. Commonly, researchers have taken to averaging samples within animals to deal with the issue of pseudo-replication (Lazic, 2010). Hierarchical modelling takes a principled approach to pseudo-replication by modelling the replication structure explicitly (Greenland, 2000). We used the HLM approach here and demonstrated an enhancement in parameter estimation.

While this data handling effectively deals with pseudo-replication, it generates a more complex than usual process for delineating the influence of image number on statistical power. The number of images captured is generally at the discretion of the researcher and commonly decided without reference to the statistical power of the experimental design. The effects of image number and sample size on statistical power were considered here (Table 4). As shown in Figure 6, image number had a profound effect on the statistical power of the experiment regardless of statistical method utilised. However, there were substantial differences between tests as to their robustness to differentiate power relative to image number. The HLM, MannWhitney $U$ test and the t-test on transformed data reached a higher level of power with fewer images compared to untransformed t-test analysis. We also demonstrated a significant beneficial effect on power can be achieved by capturing more images relative to the more expensive process of increasing the number of animals per group (see Figure 6). The beneficial effect of image number on power is good news for studies that have an exceptionally high ethical/cost burden per animal. 
The HLM on untransformed data, the t-test on transformed data and the MannWhitney $U$ test clearly outperformed the t-test carried out on the untransformed data. However, when the scale parameter was increased, the HLM method outperformed both the t-test and the Mann-Whitney $U$ test. For the intervention treatments described, power for the HLM method reached $80 \%$ for an effect size of 2.45 (Table 1). In contrast, the conventional t-test approach to data analysis, showed that much larger effect sizes were necessary $(\sim 4.18)$ to achieve $80 \%$ power. It should be noted that when both the mean and scale parameters were modified (decreased mean, increased scale), the t-test and Mann-Whitney $U$ had greatly diminished power compared to HLM. The latter emphasises the focus on mean/median differences as opposed to consideration of distribution differences. The probability of a type 1 error was not increased when using HLM methods, while for the t-test and Mann-Whitney $U$ test maintained a type I error rate of less than $0.05(0.021$ and 0.033 , respectively)

\subsection{Chi-Square distribution of the HLM test statistic}

A useful aspect of the proposed analytical framework is that there is a theoretical distribution for the generation of critical values for hypothesis testing. The likelihoodratio test used is nested and allows a direct comparison of the probability of observing either the null and alternative hypothesis (Blume, 2002). Hypothesis testing for the proposed nested hierarchical model can conveniently use a chi-square distribution with degrees of freedom equal to the difference in the number of parent parameters between the null and alternative hypothesis (Johnson \& Omland, 2004).

We compared the distribution of the likelihood-ratio test statistic using simulation and show that the distribution approximated a chi-square distribution ( $\mathrm{df}=4$; the number of 
constrained parameters; Figure 3) providing confidence in using the chi-square distribution to estimate the p-values for treatment comparisons.

Initial impressions of Figures 4 and 7 will lead the researchers to believe that the animal SD are drawn from the same distribution, regardless of group assignment. If this assumption was true, the researchers may gain extra power by not having to estimate extra parameters. As we showed above, the critical value of a likelihood ratio test is determined by the difference in degrees of freedom between the null and alternative hypothesis. Researchers should consider the influence of estimating parameters on power to detect group differences.

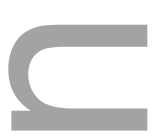

\subsection{Treatment effects}

Consideration of each respective analysis contrasting the densitometric sums for control versus treatment suggests that the treated group has increased brain parenchymal extravasation of plasma derived IgG. In the HLM model, defining the 95\% confidence intervals for distribution parameters allows explicit determination of parameters that differ. Figure 7 shows no overlap in the M parameter and relatively complete overlap in the alpha and beta parameters between the control and treatment group. Taken together, the result indicates that the treatment increased the log-normal mean with a corresponding scaled increase in variance and rightward skew when back-transformed. In a biological context, this could be interpreted as evidence for a ubiquitous, but subtle, capillary dysfunction coincident with large focal regions of dysfunction. In contrast, when only the tests for pointwise shifts in mean or median are assessed, other data elements that may be biologically relevant are over-looked. 


\subsection{Interpreting parameters}

Some of the parameters obtained from the hierarchical model may present a challenge in interpretation. While, the normal distribution has two parameters, one describing the central tendency/location (mean) and one describing the variation/scale (standard deviation) that are familiar to researchers, other distributions have more foreign parameter sets. For example, the gamma distribution is defined by a shape and scale/rate parameters and without an easily identifiable location parameter (n.b. location estimates can be made, such as: mode $=\frac{\alpha-1}{\beta}$; mean $=\frac{\alpha}{\beta}$, where $\alpha$ is the shape parameter and $\beta$ is the rate parameter, but are not at first glance intuitively accessible). As an example, two gamma distributions can be conceived with shape and rate parameters alpha $=4$, beta $=3$ respectively for the first distribution and alpha $=21$ and beta $=20$ for the second. These distributions differ in the spread, but centre near the same region. Nevertheless, these are hopefully temporary setbacks to the use of these analytic methods and distributions.

\subsection{Limitations}

Limitations of the method detailed principally revolve around the computational methods. There are abundant software programs available that are capable of performing the analysis presented in this paper. Free integrated development environments for statistical/programming languages, such as $R$ and python, enhance the availability of such methods for researchers in addition to commercial software such as Matlab. We prototyped the methods in R (R Core Team, 2015) and thereafter, ported them to $\mathrm{C}++$ for the enhanced performance. 
In order to encourage biologists to uptake hierarchical analysis, distribution fitting and power analysis, we have supplied supplementary $\mathrm{R}$ code. Distribution fitting utilises the fitdistrplus package (Delignette-Muller \& Dutang, 2015) and hierarchical analysis is performed with the Ime4 package (Bates et al., 2015). Examples of both generalized (glmer) and transformed data analysis (Imer) is shown. It should be noted that these functions do not implicitly test for differences in standard deviation, nor incorporate non-normal distributions for random effects.

A concern with the use of more complex statistical models is the problem of over fitting (Burnham \& Anderson, 1992). We utilised the Akaike Information Criterion (AIC) to penalize increases in the numbers of parameters for the estimation of the appropriate distribution. Combined with the goodness-of-fit test, we can then determine whether the distributions tested are plausible fits to the data while limiting overfitting. There was a clear consensus in both the goodness-of-fit test and AIC that a log-normal distribution best explained the data.

\section{CONCLUSION}

Appropriate distribution fitting of immunofluorescence data and the use of hierarchical modelling enhances parameter estimation, thereby strengthening the statistical analysis of immunofluorescence data. We further show through power analyses that experimental designs that are able to record a greater number of images can have significantly enhancements in power compared to studies that use few images per animal. This provides significant benefits in the context of animal ethics and resource considerations. We propose that researchers make use of hierarchical statistical models and power analysis to optimise analysis of 
immunofluorescence data, increase study design efficiency and to better characterise important biological processes.

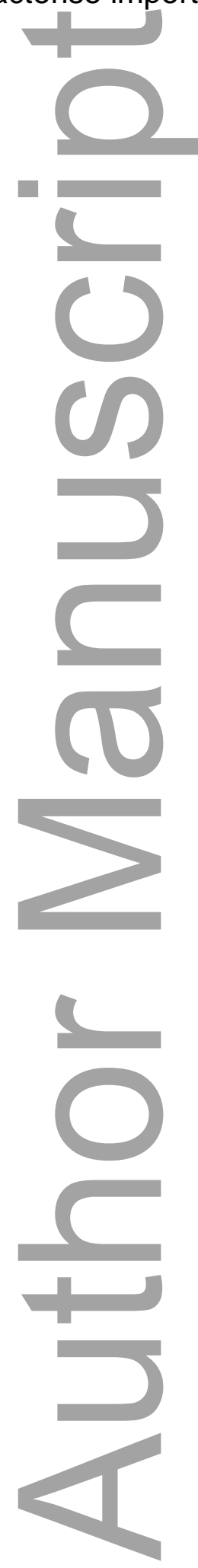




\section{Acknowledgements:}

Research was supported by the Australian National Health and Medical Research Council www.nhmrc.gov.au. Project grant 1064567. The funder had no role in study design, data collection and analysis, decision to publish, or preparation of the manuscript.

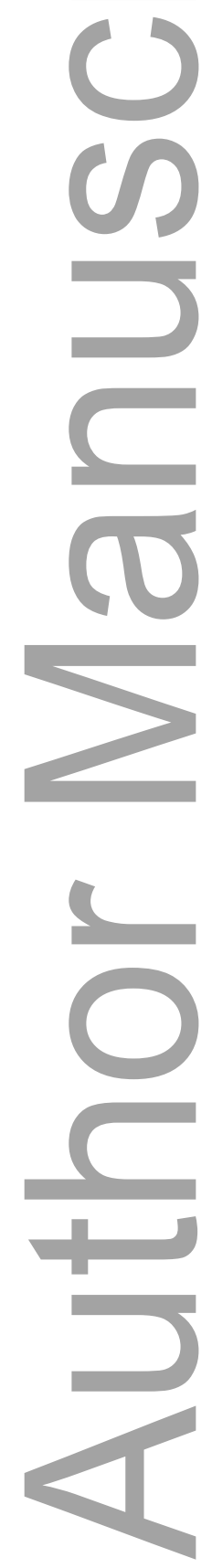


Bates, D., Machler, M., Bolker, B. M. \& Walker, S. C. (2015) Fitting Linear MixedEffects Models Using Ime4. J Stat Softw, 67, 1-48.

Blume, J. D. (2002) Likelihood methods for measuring statistical evidence. Stat Med, 21, 2563-2599.

Burnham, K. P. \& Anderson, D. R. (1992) Data-Based Selection of an Appropriate Biological Model - the Key to Modern Data-Analysis. Wildlife 2001 : Populations, 16-30.

Chinta, L. V., Lindvere, L., Dorr, A., Sahota, B., Sled, J. G. \& Stefanovic, B. (2012) Quantitative estimates of stimulation-induced perfusion response using twophoton fluorescence microscopy of cortical microvascular networks. Neuroimage, 61, 517-524.

Clauset, A., Shalizi, C. R. \& Newman, M. E. J. (2009) Power-Law Distributions in Empirical Data. Siam Rev, 51, 661-703.

Delignette-Muller, M. L. \& Dutang, C. (2015) fitdistrplus: An R Package for Fitting Distributions. J Stat Softw, 64, 1-34.

Duncan, C., Jones, K. \& Moon, G. (1998) Context, composition and heterogeneity: Using multilevel models in health research. Soc Sci Med, 46, 97-117.

Fagerland, M. W. \& Sandvik, L. (2009) Performance of five two-sample location tests for skewed distributions with unequal variances. Contemp Clin Trials, 30, 490496.

Greenland, S. (2000) Principles of multilevel modelling. Int J Epidemiol, 29, 158-167.

Handfield, L. F., Strome, B., Chong, Y. T. \& Moses, A. M. (2015) Local statistics allow quantification of cell-to-cell variability from high-throughput microscope images. Bioinformatics, 31, 940-947.

Hyman, B. T., West, H. L., Rebeck, G. W., Buldyrev, S. V., Mantegna, R. N., Ukleja, M., Havlin, S. \& Stanley, H. E. (1995) Quantitative analysis of senile plaques in Alzheimer disease: observation of log-normal size distribution and molecular epidemiology of differences associated with apolipoprotein $\mathrm{E}$ genotype and trisomy 21 (Down syndrome). Proc Natl Acad Sci U S A, 92, 3586-3590.

Ji, H. K. \& Liu, X. S. (2010) Analyzing 'omics data using hierarchical models. Nat Biotechnol, 28, 337-340.

Johnson, J. B. \& Omland, K. S. (2004) Model selection in ecology and evolution. Trends Ecol Evol, 19, 101-108.

Kitchen, C. M. R. (2009) Nonparametric versus parametric tests of location in biomedical research. American journal of ophthalmology, 147, 571-572.

Lam, V., Takechi, R., Pallabage-Gamarallage, M., Giles, C. \& Mamo, J. C. (2015) The vitamin $\mathrm{D}$, ionised calcium and parathyroid hormone axis of cerebral capillary function: therapeutic considerations for vascular-based neurodegenerative disorders. PloS one, 10, e0125504.

Lampariello, F. \& Aiello, A. (1998) Complete mathematical modeling method for the analysis of immunofluorescence distributions composed of negative and weakly positive cells. Cytometry, 32, 241-254.

Lazic, S. E. (2010) The problem of pseudoreplication in neuroscientific studies: is it affecting your analysis? BMC Neurosci, 11, 5.

Lindvere, L., Janik, R., Dorr, A., Chartash, D., Sahota, B., Sled, J. G. \& Stefanovic, B. (2013) Cerebral microvascular network geometry changes in response to functional stimulation. Neuroimage, 71, 248-259.

MacDonald, P. (1999) Power, Type I, and Type III error rates of parametric and nonparametric statistical tests. J Exp Educ, 67, 367-379. 
Manning, W. G. \& Mullahy, J. (2001) Estimating log models: to transform or not to transform? J Health Econ, 20, 461-494.

Park, C. C., Georgescu, W., Polyzos, A., Pham, C., Ahmed, K. M., Zhang, H. \& Costes, S. V. (2013) Rapid and automated multidimensional fluorescence microscopy profiling of 3D human breast cultures. Integrative biology : quantitative biosciences from nano to macro, 5, 681-691.

Parkin, T. B. (1993) Evaluation of Statistical-Methods for Determining Differences between Samples from Lognormal Populations. Agron J, 85, 747-753.

R Core Team (2015) R: A Language and Environment for Statistical Computing. 3.2.3 ed. R Foundation for Statistical Computing.

Takechi, R., Galloway, S., Pallebage-Gamarallage, M. M., Wellington, C. L., Johnsen, R. D., Dhaliwal, S. S. \& Mamo, J. C. (2010) Differential effects of dietary fatty acids on the cerebral distribution of plasma-derived apo B lipoproteins with amyloid-beta. Br J Nutr, 103, 652-662.

Vallmitjana, A., Barriga, M., Hove-Madsen, L. \& Benitez, R. (2013) Multilevel analysis of calcium dynamics in stimulated cultures of cardiomyocytes. Conference proceedings : ... Annual International Conference of the IEEE Engineering in Medicine and Biology Society. IEEE Engineering in Medicine and Biology Society. Annual Conference, 2013, 6514-6517.

Yang, G. (2013) Bioimage informatics for understanding spatiotemporal dynamics of cellular processes. Wiley interdisciplinary reviews. Systems biology and medicine, 5, 367-380.

Zhou, X. H., Gao, S. J. \& Hui, S. L. (1997) Methods for comparing the means of two independent log-normal samples. Biometrics, 53, 1129-1135.

Zimmerman, D. W. (1995) Increasing the power of nonparametric tests by detecting and downweighting outliers. J Exp Educ, 64, 71-78.

Figure 1. Structure of the hierarchical model and dependence of experimental sampling. The parent distribution parameters $M$ and $S$ represent the mean and SD of the group, which in turn inform the subject level means $\mu$. The parent distribution parameters $\alpha$ and $\beta$ are the shape and rate parameters of the gamma distribution that then informs the subject level precisions $\phi$.

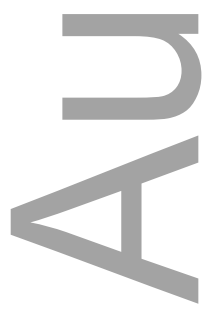




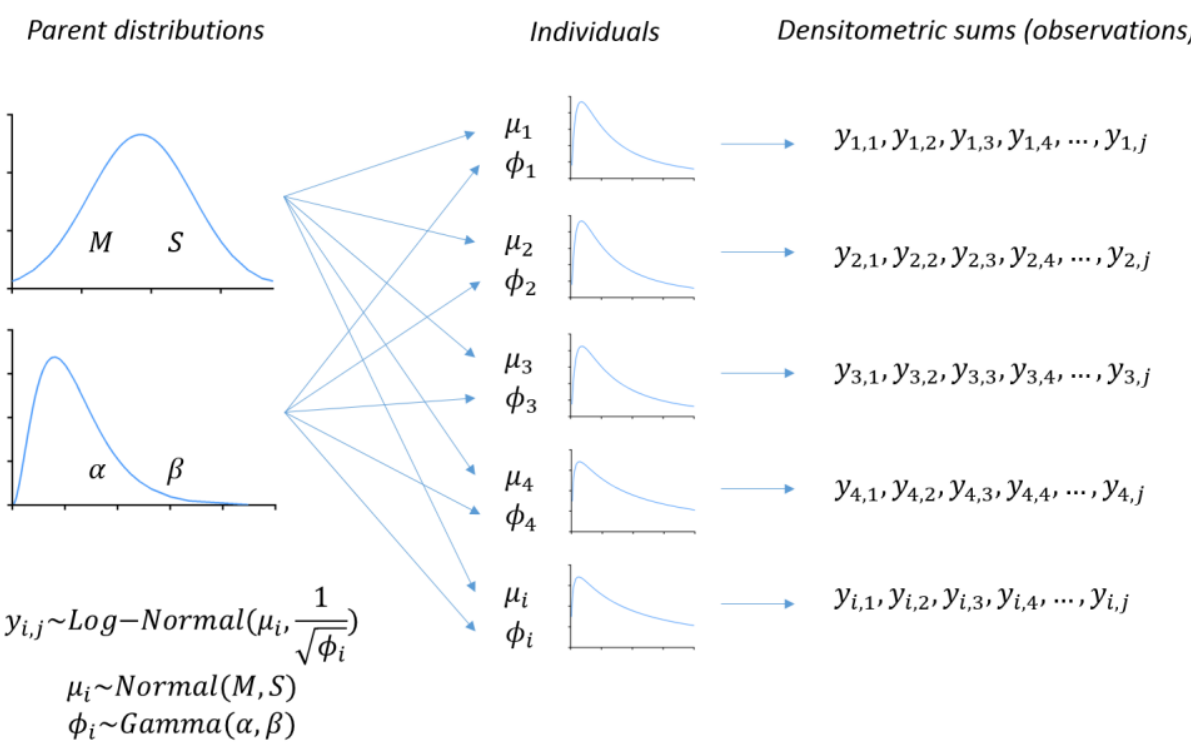

Figure 2. Empirical cumulative density function and representative immunofluorescence micrographs and boxplots of Kolmogorov-Smirnov test statistics for goodness-of-fit. As can be seen from the left and right panels, the data are clearly non-normal and likely best represented by a log-normal distribution.

(Scale bar $=30 \mu \mathrm{m})$
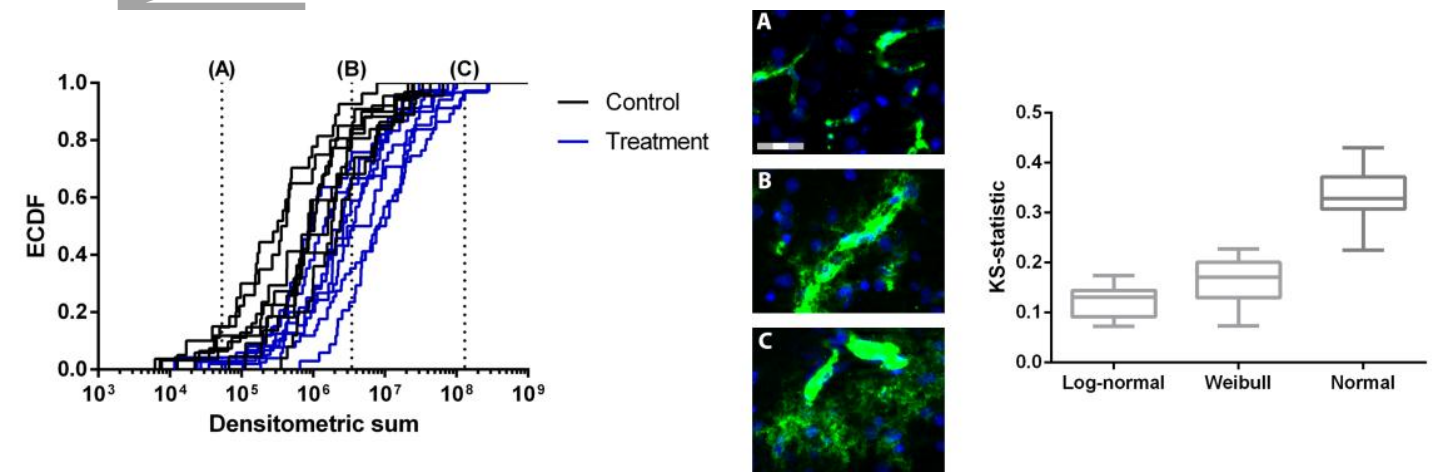

Figure 3. Comparison of the likelihood ratio test statistic distribution against the Chisquare distribution and critical values. Our empirically derived distribution can be seen to follow very closely the Chi-square distribution with $\mathrm{df}=4$. 


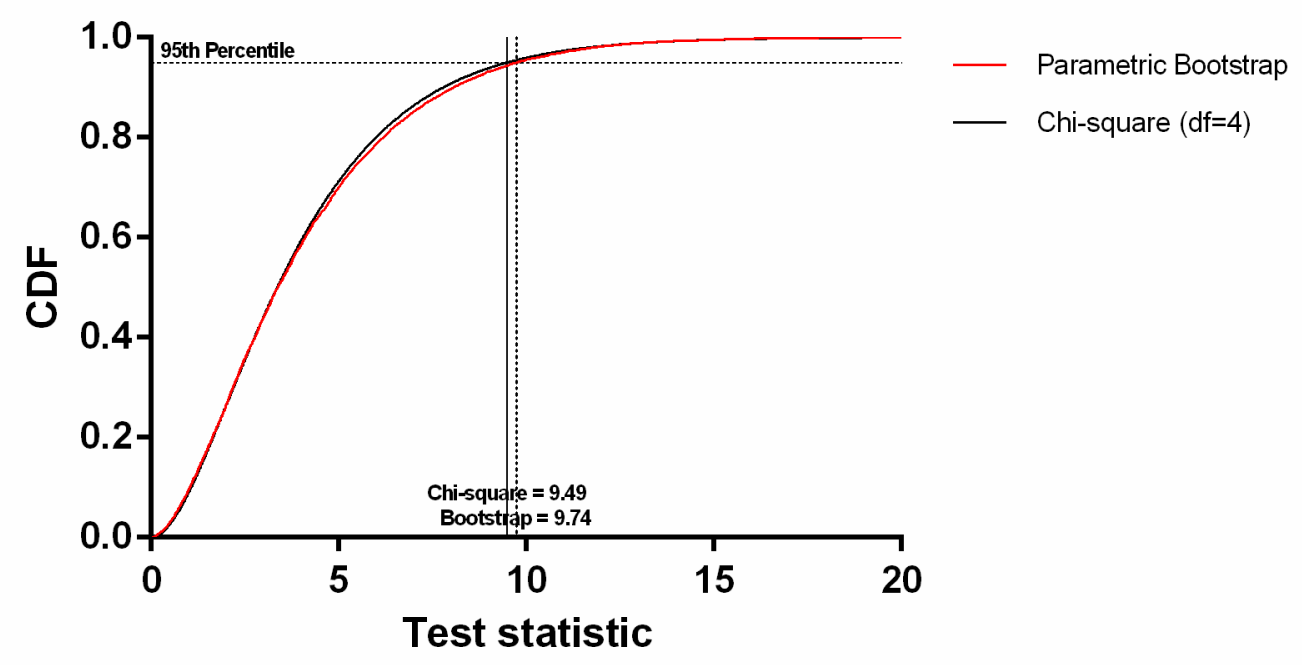

Figure 4. Individual animal parameter estimates $(+95 \% \mathrm{Cl}$; horizontal bars) and confidence intervals (grey area) for log-normal mean (mu) and standard deviation (sigma) under the null and alternative hypothesis derived from the hierarchical model. The non-overlapping vertical grey bars for the alternative hypothesis mean estimates (bottom left) indicate a significant increase in extravasation in the treated group, but that this did not correspond with an increase in SD (bottom right). 
MU
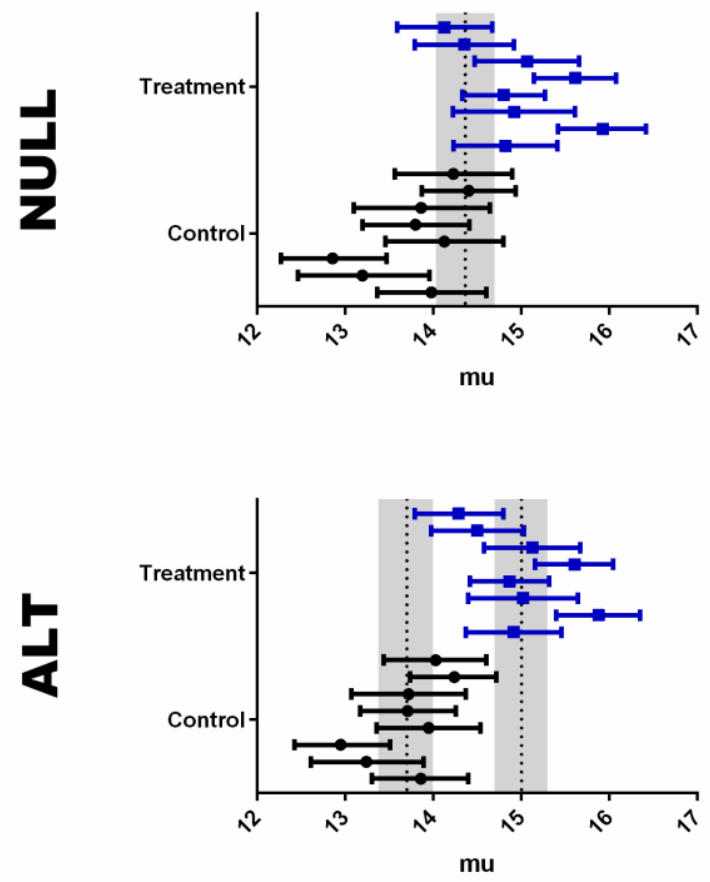

SIGMA
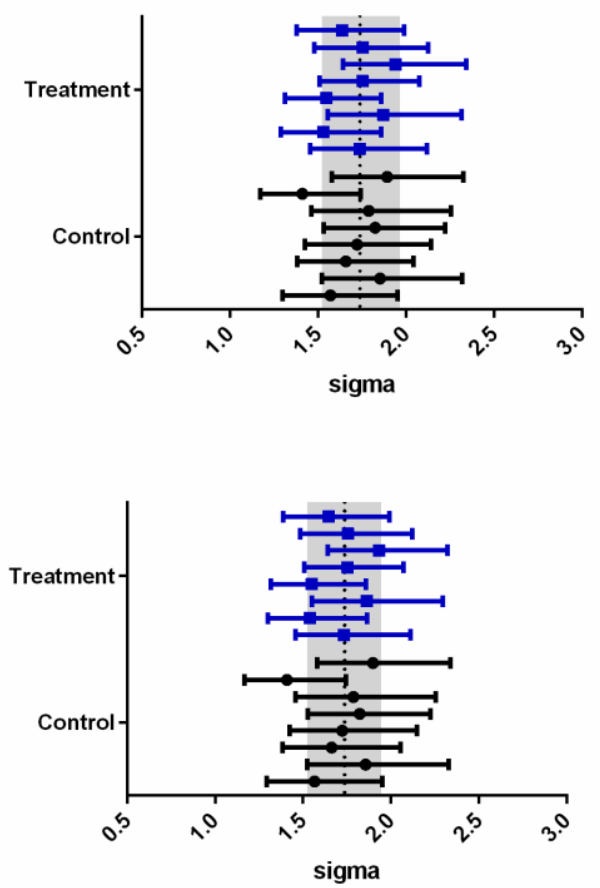

Figure 5. Untransformed and log-transformed animal densitometric sums with bootstrapped confidence intervals (horizontal bars), standard deviation parameter estimates and confidence intervals (grey area). 


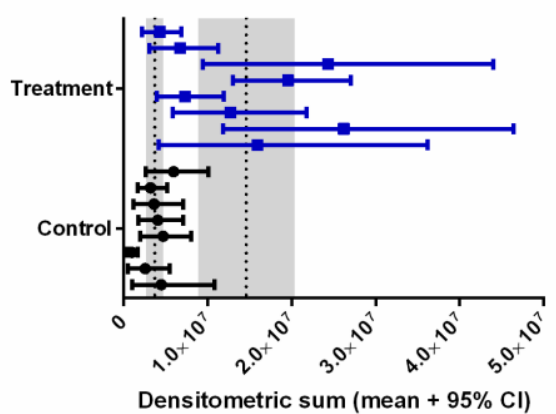

Densitometric sum (mean $+95 \% \mathrm{Cl}$ )

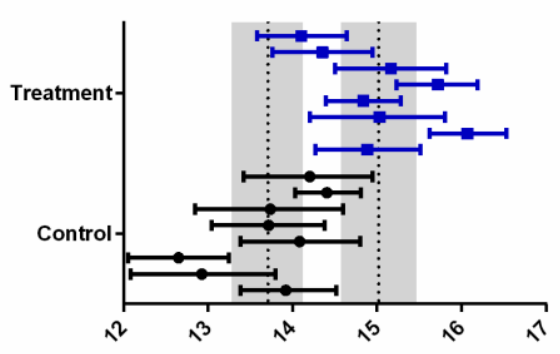

Log transformed densitometric sum (mean $+95 \% \mathrm{Cl}$ )
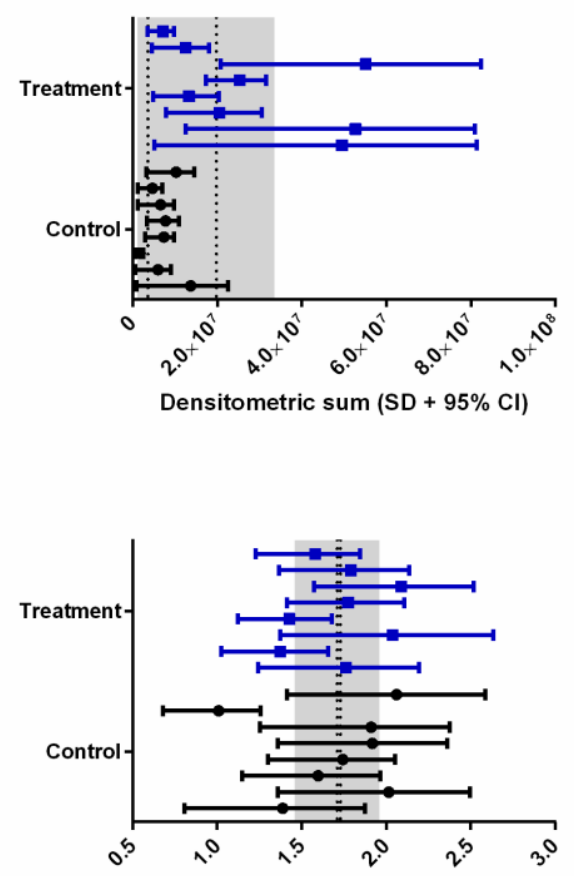

Log transformed densitometric sum (SD + 95\% CI)

Figure 6. Statistical power for combinations of animals (4-12) and images (5-80) for a given effect size of 2.45. The overall pattern shows the expected increase in power when more animals and images are used.
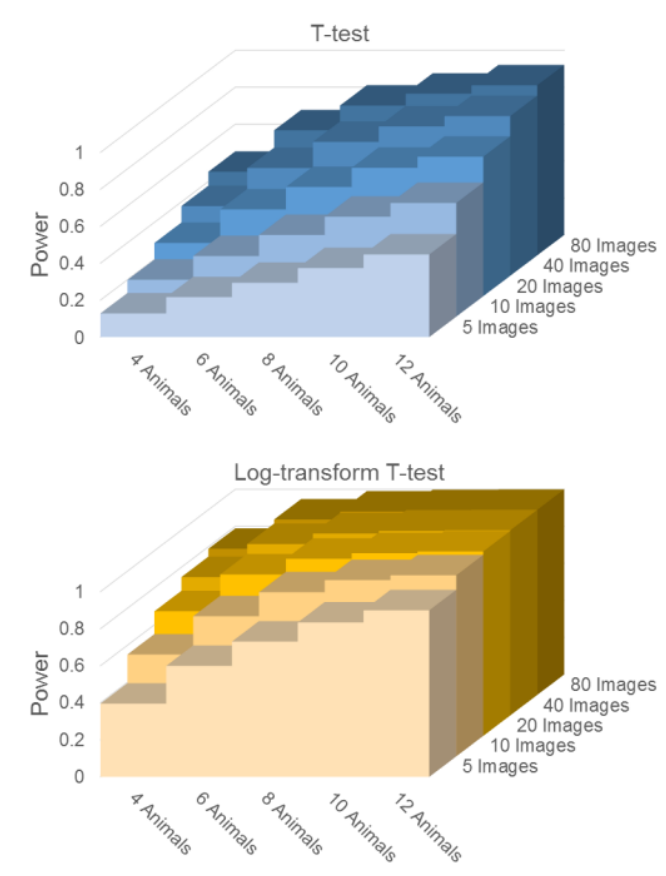
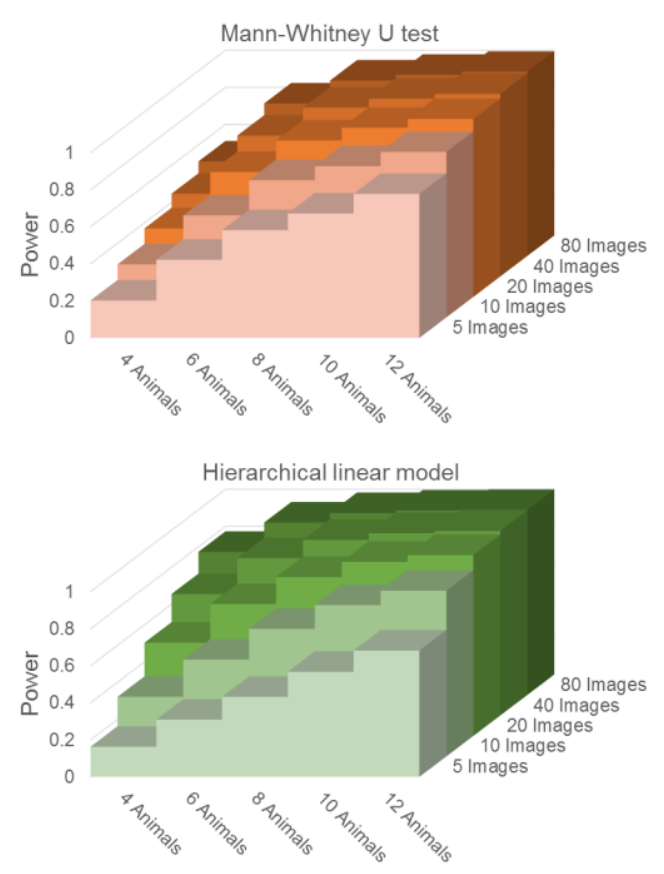
Figure 7. 95 percent confidence bands for parent distribution parameters under the alternative hypothesis. Left) Parent distribution means (M) and SDs (S) that describe the distribution of subject level means. The heteroscedastic distribution of means along SD is an expected response due to increased/decreased 'freedom' to explain means according to changes in SD, at the expense of likelihood. Right) Parent distribution shape (Alpha) and rate (Beta) parameter estimates that describe the distribution of subject level SDs.
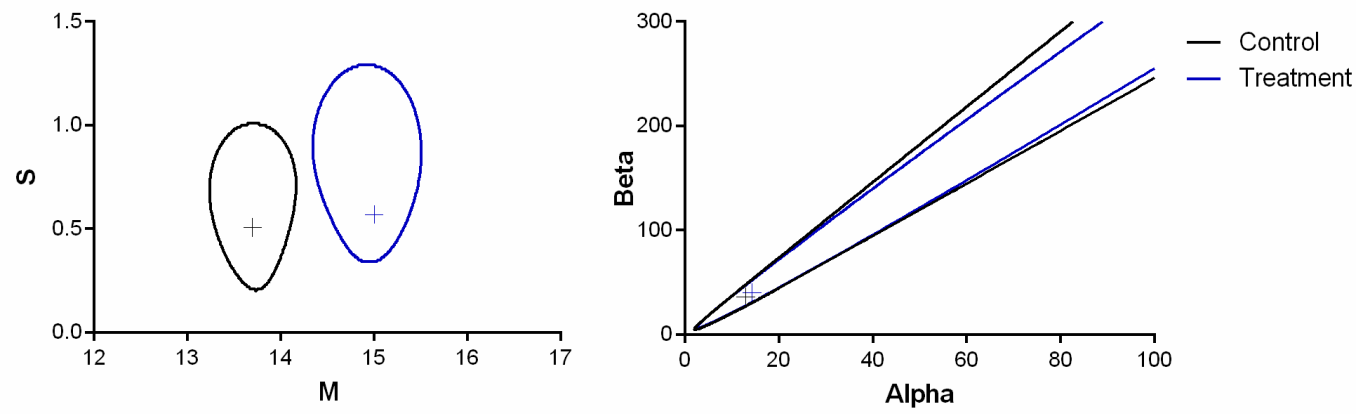

Table 1. Parent distribution parameter sets used for determination of Type I and II power. All parameter sets were compared against another group assigned the parent distribution parameters from the control group $(M=13.70$; $S=0.51$; alpha=12.93; beta=35.95).

\begin{tabular}{|c|c|c|c|c|c|c|c|c|}
\hline \multirow[t]{2}{*}{ M } & & \multirow[t]{2}{*}{ alpha } & \multirow[t]{2}{*}{ Beta } & \multirow[t]{2}{*}{ T-test } & \multirow{2}{*}{$\begin{array}{l}\text { Log } \\
\text { transfor } \\
\text { m t-test }\end{array}$} & \multirow{2}{*}{$\begin{array}{l}\text { Mann } \\
\text { Whitney } \\
\text { U }\end{array}$} & \multicolumn{2}{|c|}{ HLM } \\
\hline & & & & & & & $\begin{array}{c}\text { Chi- } \\
\text { square }\end{array}$ & $\begin{array}{l}\text { Monte } \\
\text { carlo }\end{array}$ \\
\hline 13.7 & 0.51 & 12.93 & 35.95 & 0.021 & 0.051 & 0.033 & 0.056 & $0.050^{*}$ \\
\hline 13.9 & 0.51 & 12.93 & 35.95 & 0.052 & 0.103 & 0.084 & 0.096 & 0.087 \\
\hline 14.1 & 0.51 & 12.93 & 35.95 & 0.111 & 0.253 & 0.183 & 0.197 & 0.185 \\
\hline 14.3 & 0.51 & 12.93 & 35.95 & 0.203 & 0.491 & 0.334 & 0.367 & \begin{tabular}{|l|}
0.349 \\
\end{tabular} \\
\hline 14.5 & 0.51 & 12.93 & 35.95 & 0.320 & 0.729 & 0.505 & 0.575 & 0.558 \\
\hline 14.7 & 0.51 & 12.93 & 35.95 & 0.443 & 0.897 & 0.677 & 0.765 & 0.749 \\
\hline 14.9 & 0.51 & 12.93 & 35.95 & 0.557 & 0.969 & 0.813 & 0.900 & 0.891 \\
\hline 15.1 & 0.51 & 12.93 & 35.95 & 0.654 & 0.993 & 0.903 & 0.963 & 0.959 \\
\hline
\end{tabular}




\begin{tabular}{|l|l|l|l|l|l|l|l|l|}
\hline 15.3 & 0.51 & 12.93 & 35.95 & 0.729 & 0.999 & 0.957 & 0.991 & 0.989 \\
\hline 15.5 & 0.51 & 12.93 & 35.95 & 0.782 & 1.000 & 0.980 & 0.998 & 0.997 \\
\hline 15.7 & 0.51 & 12.93 & 35.95 & 0.821 & 1.000 & 0.992 & 1.000 & 1.000 \\
\hline 13.7 & 0.51 & 17.14 & 60.50 & 0.061 & 0.050 & 0.106 & 0.157 & 0.144 \\
\hline 13.7 & 0.51 & 20.78 & 90.18 & 0.149 & 0.051 & 0.279 & 0.438 & 0.420 \\
\hline 13.7 & 0.51 & 24.86 & 129.9 & 0.256 & 0.052 & 0.497 & 0.764 & 0.750 \\
\hline 13.7 & 0.51 & 29.24 & 181.2 & 0.359 & 0.054 & 0.708 & 0.932 & 0.925 \\
\hline 13.7 & 0.51 & 33.99 & 246.1 & 0.419 & 0.054 & 0.855 & 0.987 & 0.984 \\
\hline 13.7 & 0.51 & 39.16 & 327.9 & 0.454 & 0.055 & 0.938 & 0.998 & 0.998 \\
\hline 13.5 & 0.51 & 17.14 & 60.50 & 0.026 & $0.094^{* *}$ & 0.045 & 0.190 & 0.176 \\
\hline 13.3 & 0.51 & 20.78 & 90.18 & 0.039 & $0.237^{* *}$ & 0.071 & 0.568 & 0.547 \\
\hline 13.1 & 0.51 & 24.86 & 129.9 & 0.056 & $0.461^{* *}$ & 0.105 & 0.892 & 0.883 \\
\hline 12.9 & 0.51 & 29.24 & 181.2 & 0.079 & $0.664^{* *}$ & 0.151 & 0.986 & 0.984 \\
\hline
\end{tabular}

${ }^{*}$ Monte carlo generation of critical value would mean this is equal to alpha (0.05)

${ }^{* *}$ Power to detect changes in the distribution median (decreasing) and not changes in the distribution mean (increasing).

Table 2. Individual animal goodness-of-fit and distribution fitting results. AIC = Akaike information criterion. $\mathrm{KS}$ statistic $=$ Kolmogorov-Smirnov statistic. $\mathrm{P}$-value $=$ percentage of bootstrap replicates with test statistic larger than measured. $C T R L=$ Control animal. TREAT $=$ Treatment animal.

Model fit: Log-normal distribution

\begin{tabular}{|l|l|l|l|l|l|l|l|}
\hline Animal & AIC & $\begin{array}{l}\text { K-S } \\
\text { statistic }\end{array}$ & p-value & Animal & AIC & $\begin{array}{l}\text { K-S } \\
\text { statistic }\end{array}$ & $p$-value \\
\hline CTRL 1 & 692 & 0.14 & 0.30 & TREAT 1 & 1015 & 0.14 & 0.17 \\
\hline CTRL 2 & 605 & 0.12 & 0.59 & TREAT 2 & 1214 & 0.08 & 0.82 \\
\hline CTRL 3 & 788 & 0.09 & 0.88 & TREAT 3 & 827 & 0.13 & 0.40 \\
\hline CTRL 4 & 710 & 0.17 & 0.08 & TREAT 4 & 1299 & 0.10 & 0.38 \\
\hline CTRL 5 & 982 & 0.14 & 0.13 & TREAT 5 & 1810 & 0.07 & 0.72 \\
\hline
\end{tabular}




\begin{tabular}{|l|l|l|l|l|l|l|l|}
\hline CTRL 6 & 540 & 0.15 & 0.45 & TREAT 6 & 1285 & 0.11 & 0.36 \\
\hline CTRL 7 & 795 & 0.11 & 0.60 & TREAT 7 & 1115 & 0.08 & 0.85 \\
\hline CTRL 8 & 886 & 0.16 & 0.10 & TREAT 8 & 1058 & 0.15 & 0.06 \\
\hline
\end{tabular}

Model fit: Normal distribution

\begin{tabular}{|l|l|l|l|l|l|l|l|}
\hline Animal & AIC & $\begin{array}{l}\text { K-S } \\
\text { statistic }\end{array}$ & $p$-value & Animal & AIC & $\begin{array}{l}\text { K-S } \\
\text { statistic }\end{array}$ & $p$-value \\
\hline CTRL 1 & 789 & 0.43 & 0.00 & TREAT 1 & 1151 & 0.38 & 0.00 \\
\hline CTRL 2 & 684 & 0.37 & 0.00 & TREAT 2 & 1308 & 0.35 & 0.00 \\
\hline CTRL 3 & 855 & 0.31 & 0.00 & TREAT 3 & 879 & 0.27 & 0.00 \\
\hline CTRL 4 & 761 & 0.32 & 0.00 & TREAT 4 & 1393 & 0.29 & 0.00 \\
\hline CTRL 5 & 1075 & 0.41 & 0.00 & TREAT 5 & 1886 & 0.23 & 0.00 \\
\hline CTRL 6 & 585 & 0.34 & 0.00 & TREAT 6 & 1427 & 0.37 & 0.00 \\
\hline CTRL 7 & 842 & 0.33 & 0.00 & TREAT 7 & 1211 & 0.31 & 0.00 \\
\hline CTRL 8 & 951 & 0.33 & 0.00 & TREAT 8 & 1138 & 0.31 & 0.00 \\
\hline
\end{tabular}

Model fit: Weibull distribution

\begin{tabular}{|l|l|l|l|l|l|l|l|}
\hline Animal & AIC & $\begin{array}{l}\text { K-S } \\
\text { statistic }\end{array}$ & $p$-value & Animal & AIC & $\begin{array}{l}\text { K-S } \\
\text { statistic }\end{array}$ & -value \\
\hline CTRL 1 & 703 & 0.21 & 0.03 & TREAT 1 & 1023 & 0.19 & 0.02 \\
\hline CTRL 2 & 609 & 0.19 & 0.11 & TREAT 2 & 1223 & 0.16 & 0.05 \\
\hline CTRL 3 & 791 & 0.17 & 0.10 & TREAT 3 & 825 & 0.13 & 0.44 \\
\hline CTRL 4 & 713 & 0.23 & 0.01 & TREAT 4 & 1305 & 0.14 & 0.08 \\
\hline CTRL 5 & 985 & 0.21 & 0.01 & TREAT 5 & 1805 & 0.07 & 0.75 \\
\hline CTRL 6 & 541 & 0.12 & 0.80 & TREAT 6 & 1289 & 0.17 & 0.01 \\
\hline CTRL 7 & 802 & 0.18 & 0.07 & TREAT 7 & 1119 & 0.15 & 0.07 \\
\hline CTRL 8 & 884 & 0.12 & 0.45 & TREAT 8 & 1064 & 0.20 & 0.01 \\
\hline
\end{tabular}


Table 3. Maximum likelihood estimates of parent distribution parameters and negative log likelihoods of null and alternative hypothesis.

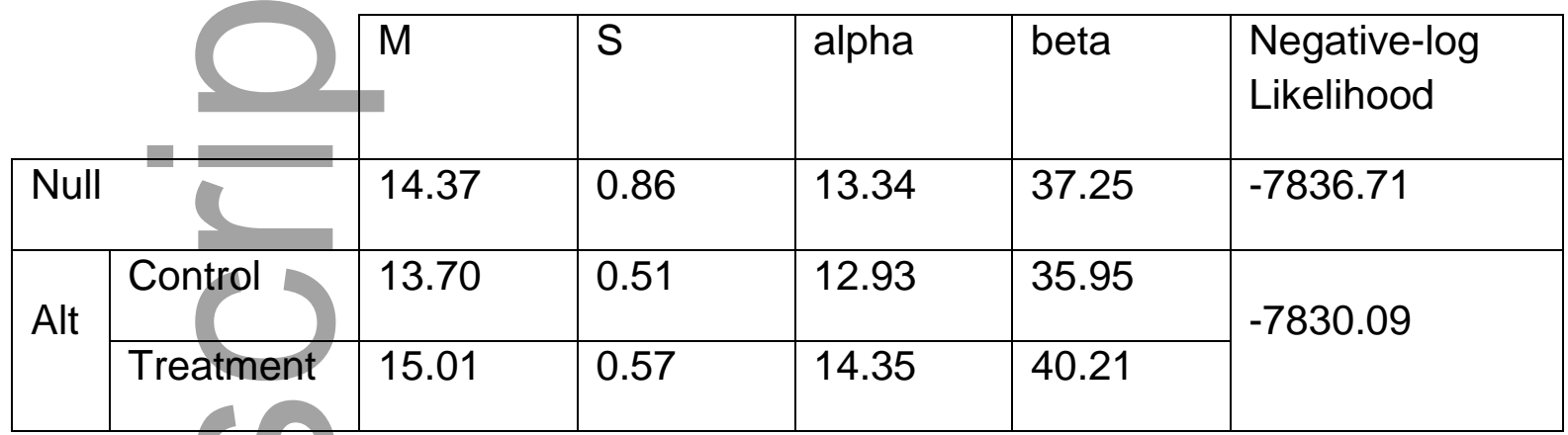

Table 4. Power analysis for given combinations of sample size and images captured per sample. Parent distribution parameters for the two groups were those obtained under the alternative hypothesis $(M=13.7, S=0.51$, alpha $=12.93$, beta $=35.95$ vs $M=15.01, S=0.57$, alpha $=14.35$, beta $=40.21$ ).

HLM - Monte Carlo (critical value=9.74)

\begin{tabular}{|l|l|l|l|l|l|}
\cline { 2 - 6 } \multicolumn{1}{c|}{} & 5 Images & 10 Images & 20 Images & 40 Images & 80 Images \\
\hline 4 Animals & 0.163 & 0.322 & 0.504 & 0.656 & 0.773 \\
\hline 6 Animals & 0.309 & 0.523 & 0.712 & 0.849 & 0.931 \\
\hline 8 Animals & 0.432 & 0.689 & 0.858 & 0.948 & 0.983 \\
\hline 10 Animals & 0.567 & 0.817 & 0.940 & 0.984 & 0.996 \\
\hline 12 Animals & 0.682 & 0.895 & 0.976 & 0.997 & 0.999 \\
\hline
\end{tabular}

T-test

\begin{tabular}{|l|l|l|l|l|l|}
\cline { 2 - 6 } \multicolumn{1}{l|}{} & 5 Images & 10 Images & 20 Images & 40 Images & 80 Images \\
\hline 4 Animals & 0.128 & 0.200 & 0.289 & 0.378 & 0.453 \\
\hline 6 Animals & 0.216 & 0.328 & 0.470 & 0.583 & 0.677 \\
\hline 8 Animals & 0.294 & 0.443 & 0.592 & 0.725 & 0.811 \\
\hline 10 Animals & 0.374 & 0.541 & 0.695 & 0.809 & 0.876 \\
\hline
\end{tabular}




\begin{tabular}{|l|l|l|l|l|l|}
\hline 12 Animals & 0.448 & 0.616 & 0.756 & 0.865 & 0.921 \\
\hline
\end{tabular}

Log-transform T-test

\begin{tabular}{|l|l|l|l|l|l|}
\cline { 2 - 6 } \multicolumn{1}{c|}{} & 5 Images & 10 Images & 20 Images & 40 Images & 80 Images \\
\hline 4 Animals & 0.396 & 0.550 & 0.673 & 0.747 & 0.789 \\
\hline 6 Animals & 0.599 & 0.757 & 0.872 & 0.926 & 0.948 \\
\hline 8 Animals & 0.731 & 0.886 & 0.957 & 0.982 & 0.990 \\
\hline 10 Animals & 0.834 & 0.952 & 0.987 & 0.996 & 0.998 \\
\hline 12 Animals & 0.900 & 0.978 & 0.996 & 0.999 & 1.000 \\
\hline
\end{tabular}

Mann-Whitney U-test

\begin{tabular}{|l|l|l|l|l|l|}
\cline { 2 - 6 } \multicolumn{1}{l|}{} & 5 Images & 10 Images & 20 Images & 40 Images & 80 Images \\
\hline 4 Animals & 0.202 & 0.286 & 0.370 & 0.447 & 0.511 \\
\hline 6 Animals & 0.422 & 0.551 & 0.675 & 0.761 & 0.824 \\
\hline 8 Animals & 0.582 & 0.741 & 0.845 & 0.912 & 0.947 \\
\hline 10 Animals & 0.672 & 0.817 & 0.914 & 0.960 & 0.978 \\
\hline 12 Animals & 0.777 & 0.894 & 0.960 & 0.986 & 0.995 \\
\hline
\end{tabular}




\section{University Library}

\section{- M M N E R VA A gateway to Melbourne's research publications}

Minerva Access is the Institutional Repository of The University of Melbourne

Author/s:

Giles, C;Albrecht, MA;Lam, V;Takechi, R;Mamo, JC

Title:

Biostatistical analysis of quantitative immunofluorescence microscopy images

Date:

2016-12-01

Citation:

Giles, C., Albrecht, M. A., Lam, V., Takechi, R. \& Mamo, J. C. (2016). Biostatistical analysis of quantitative immunofluorescence microscopy images. JOURNAL OF MICROSCOPY, 264 (3), pp.321-333. https://doi.org/10.1111/jmi.12446.

Persistent Link:

http://hdl.handle.net/11343/291979 